\title{
Relations on higher dimensional Padovan sequences
}

\section{Renata Passos Machado Vieira ${ }^{1}$, Francisco Regis Vieira Alves ${ }^{2}$ and Paula Maria Machado Cruz Catarino ${ }^{3}$}

${ }^{1}$ Department of Mathematics, Federal Institute of Education, Science and Technology of Ceará (IFCE) Fortaleza-CE, Brazil e-mail: re.passosmegmail.com

${ }^{2}$ Department of Mathematics, Federal Institute of Education, Science and Technology of Ceará (IFCE) Fortaleza-CE, Brazil e-mail: fregis@gmx.fr

${ }^{3}$ Department of Mathematic, University of Trás-os-Montes and Alto Douro Vila Real, Portugal e-mail: pcatarino23@gmail.com

Abstract: Many papers developed so far for Padovan sequences properties and its extensions usually follow the one-dimensional approach. The presented work introduces new relations for a higher dimensional sequence, this approach is adopted for two, three and $n$-dimensional Padovan Sequence. Several mathematical properties are discussed for the first time in the present work. Keywords: Relation, Dimensional, Padovan sequence, Recurrent relations, Two-dimensional. 2020 Mathematics Subject Classification: 11B37, 11B39.

\section{Introduction}

The Italian architect Richard Padovan (1935 -) discovered a kind of "cousin" of the well-known Fibonacci sequence, the Padovan numbers, which are also recursive, arithmetic and linear. Richard Padovan was born in the city of Padua [17], a village a little far from the same origin of Fibonacci [6]. 
This sequence has many interesting properties and applications in fields of architecture (see, e.g., Padovan [14], Voet and Schoonjans [18]). The Padovan sequence $P(n)$ is defined by the following recurrence relation:

$$
P(n)=P(n-2)+P(n-3)
$$

where $P(0)=P(1)=P(2)=1$.

In Oliveira, Alves, and Paiva [12] and introduced by Özdemir [13], we can observe a mathematical process of extending the Fibonacci numbers in the complex plane. The method used by Oliveira, Alves and Paiva [12] allows an in the deep description of two, three and higher dimensional relations recurrence. Also in Catarino [4] studies of $k$-Pell-Lucas polynomial relation sequence contributed to this paper on introducing properties in complex space also applied here.

In a nutshell, the two-dimensional Padovan sequence elements are defined as $a+b i$, where $a$ and $b$ are the uni-dimensional padovan sequence terms, fitting the general notation described in the form $P(n)=P(n-2)+P(n-3)$. $i$, for an index $n \in \mathbb{N}$, and $i$ is the imaginary unit, where $i^{2}=-1$. Such definition introduced in this work is followed and applied to higher dimensional Padovan sequences.

These new higher dimensional Padovan definitions can be applied in areas like quantic physics, cinematics, and computer science [9].

In the following sections, we will employ such a method for the particular case of the Padovan sequence, originally only in a one-dimensional sequence.

\section{Two-dimensional recurrence for the Padovan sequence}

In this section, some two-dimensional Padovan sequence relations and properties are discussed. Some of the properties were based on Bilgici [3] who identified and proposed the 16-dimension Fibonacci and Lucas Sequences.

Definition 2.1. The numbers in the form $P(n, m)$ represent the two-dimensional Padovan sequence and must satisfy the following recurrence conditions:

$$
\left\{\begin{array}{l}
P(n+2, m)=P(n, m)+P(n-1, m) \\
P(n, m+2)=P(n, m)+P(n, m-1)
\end{array}\right.
$$

with the following initial values obtained by empirical means: $P(0,0)=1, P(1,0)=1$, $P(2,0)=1, P(0,1)=1+i, P(0,2)=1+i, P(1,1)=1+i, P(1,2)=1+i, P(2,1)=1+i$, $P(2,2)=1+i$, where $i^{2}=-1$ and $P(0)=P(1)=P(2)=1, P(-1)=0$.

The table below represents the coordinate plane of the two-dimensional Padovan relations, for any $n \times m$. 


\begin{tabular}{|c|c|c|c|c|c|c|}
\hline $\mathbf{5}$ & $3+2 i$ & $3+2 i$ & $3+2 i$ & $6+4 i$ & $6+4 i$ & $9+6 i$ \\
\hline $\mathbf{4}$ & $2+2 i$ & $2+2 i$ & $2+2 i$ & $4+4 i$ & $4+4 i$ & $6+6 i$ \\
\hline $\mathbf{3}$ & $2+i$ & $2+i$ & $2+i$ & $4+2 i$ & $4+2 i$ & $6+3 i$ \\
\hline $\mathbf{2}$ & $1+i$ & $1+i$ & $1+i$ & $2+2 i$ & $2+2 i$ & $3+3 i$ \\
\hline $\mathbf{1}$ & $1+i$ & $1+i$ & $1+i$ & $2+2 i$ & $2+2 i$ & $3+3 i$ \\
\hline $\mathbf{0}$ & 1 & 1 & 1 & 2 & 2 & 3 \\
\hline $\boldsymbol{n} \times \boldsymbol{m}$ & $\mathbf{0}$ & $\mathbf{1}$ & $\mathbf{2}$ & $\mathbf{3}$ & $\mathbf{4}$ & $\mathbf{5}$ \\
\hline
\end{tabular}

Table 1. Two-dimensional Padovan plane for initial values

Lemma 2.2. The following properties are valid:

(a) $P(n, 0)=P(n)$,

(b) $P(0, m)=P(m)+P(m-1) i$,

(c) $P(n, 1)=P(n)+P(n) i$,

(d) $P(1, m)=P(m)+P(m-1) i$.

Proof. (a) Once $P(n+2, m)=P(n, m)+P(n-1, m)$ and the initial values are $P(0,0)=1=P(0)$, $P(1,0)=1=P(1), P(2,0)=1=P(2)$. Applying the second principle of finite induction on $n$, setting $m=0$ and varying $n=0,1,2, \ldots$ One can obtain that:

$$
\begin{aligned}
P(n+2,0) & =P(n, 0)+P(n-1,0) ; \\
P(3,0) & =P(1,0)+P(0,0)=2=P(3) ; \\
P(4,0) & =P(2,0)+P(1,0)=2=P(4) ; \\
P(5,0) & =P(3,0)+P(2,0)=3=P(5) ; \\
P(6,0) & =P(4,0)+P(3,0)=4=P(6) ; \\
& \vdots \\
P(n-3,0) & =P(n-5,0)+P(n-6,0)=P(n-3) ; \\
P(n-2,0) & =P(n-4,0)+P(n-5,0)=P(n-2) ; \\
P(n-1,0) & =P(n-3,0)+P(n-4,0)=P(n-1) ; \\
P(n, 0) & =P(n-2,0)+P(n-3,0)=P(n-2)+P(n-3)=P(n) .
\end{aligned}
$$

The other identities are demonstrated similarly to identity (a).

Theorem 2.3. For $n, m \in \mathbb{N}$, the numbers in the form $P(n, m)$ are described by:

$$
P(n, m)=(P(n) P(m))+(P(n) P(m-1)) i .
$$

Proof. Let $n \in \mathbb{N}$, performing the second induction principle on $m$, for the value of $m=0$ and $m=1$, there are respectively the properties $P(n, 0)=P(n) P(1)+P(n) P(-1) i=P(n)$ and $P(n, 1)=P(n) P(1)+P(n) P(0) i=P(n)+P(n) i$ previously validated by the Lemma 2.2 , where $P(-1)=0, P(0)=P(1)=P(2)=1$, whose initial values are $P(0,0)=1, P(1,0)=1$, $P(2,0)=1, P(0,1)=1+i, P(1,1)=1+i, P(2,1)=1+i, P(0,2)=1+i, P(1,2)=1+i$, $P(2,2)=1+i$. 
For $P(n, 2)$, using the recurrence $P(n, m+2)=P(n, m)+P(n, m-1)$ with the initial values $P(0,0)=1=P(0), P(1,0)=1=P(1), P(2,0)=1=P(2), P(0,2)=1+i, P(1,2)=1+i$, $P(2,2)=1+i$, with $m=2$ fixed and $n=0,1,2,3, \ldots$, we have:

$$
\begin{aligned}
P(n+2,2) & =P(n, 2)+P(n-1,2) ; \\
P(3,2) & =P(1,2)+P(0,2)=2+2 i=P(3)+P(3) i ; \\
P(4,2) & =P(2,2)+P(1,2)=2+2 i=P(4)+P(4) i ; \\
P(5,2) & =P(3,2)+P(2,2)=3+3 i=P(5)+P(5) i ; \\
P(6,2) & =P(4,2)+P(3,2)=4+4 i=P(6)+P(6) i ; \\
& \vdots \\
P(n-3,2) & =P(n-5,2)+P(n-6,2)=P(n-3)+P(n-3) i ; \\
P(n-2,2) & =P(n-4,2)+P(n-5,2)=P(n-2)+P(n-2) i ; \\
P(n-1,2) & =P(n-3,2)+P(n-4,2)=P(n-1)+P(n-1) i ; \\
P(n, 2) & =P(n-2,2)+P(n-3,2) \\
& =P(n-2)+P(n-2) i+P(n-3)+P(n-3) i \\
& =P(n)+P(n) i .
\end{aligned}
$$

For $m=3$, we have:

$$
\begin{aligned}
P(n+2,3) & =P(n, 3)+P(n-1,3) ; \\
P(3,3) & =P(1,3)+P(0,3)=4+2 i=2 P(3)+P(3) i ; \\
P(4,3) & =P(2,3)+P(1,3)=4+2 i=2 P(4)+P(4) i ; \\
P(5,3) & =P(3,3)+P(2,3)=6+3 i=2 P(5)+P(5) i ; \\
P(6,3) & =P(4,3)+P(3,3)=8+4 i=2 P(6)+P(6) i ; \\
& \vdots \\
P(n-3,3) & =P(n-5,3)+P(n-6,3)=2 P(n-3)+P(n-3) i ; \\
P(n-2,3) & =P(n-4,3)+P(n-5,3)=2 P(n-2)+P(n-2) i ; \\
P(n-1,3) & =P(n-3,3)+P(n-4,3)=2 P(n-1)+P(n-1) i ; \\
P(n, 3) & =P(n-2,3)+P(n-3,3) \\
& =2 P(n-2)+P(n-2) i+2 P(n-3)+P(n-3) i \\
& =2 P(n)+P(n) i .
\end{aligned}
$$

Therefore, for $m=1,2, \ldots, k-2$, the following identities are valid:

$$
\begin{aligned}
P(n, 0) & =P(n) 1+P(n) 0 i=P(n) P(0)+P(n) P(-1) i ; \\
P(n, 1) & =P(n) 1+P(n) 1 i=P(n) P(1)+P(n) P(0) i ; \\
P(n, 2) & =P(n) 1+P(n) 1 i=P(n) P(2)+P(n) P(1) i ; \\
P(n, 3) & =P(n) 2+P(n) 1 i=P(n) P(3)+P(n) P(2) i ; \\
& \vdots \\
P(n, k-3) & =P(n) P(k-3)+P(n) P(k-4) i \\
P(n, k-2) & =P(n) P(k-2)+P(n) P(k-3) i .
\end{aligned}
$$


Validating for $m=k$, from recurrence $P(n, k)=P(n, k-2)+P(n, k-3)$, one can obtain:

$$
\begin{aligned}
P(n, k) & =P(n, k-2)+P(n, k-3) \\
P(n, k) & =P(n) P(k-2)+P(n) P(k-3) i+P(n) P(k-3)+P(n) P(k-4) i \\
& =P(n) P(k)+P(n) P(k-1) i .
\end{aligned}
$$

\section{Three-dimensional recurrence for the Padovan sequence}

According with Harman [7], where it explains the numbers denominated by $P(n, m)$ represent the set of integers Gaussians $(n, m)=n+m . i$, where $n$ and $m$ integers. Thus, in order to verify this extension of Padovan sequence for complex dimensions, the three-dimensional recursion relations case will be explained for the Padovan sequence.

Definition 3.1. For the Padovan sequence, considering the following initial values, defined as: $P(0,0,0)=1=P(0), P(1,0,0)=1=P(1), P(2,0,0)=1=P(2), P(0,1,0)=1+i$, $P(0,0,1)=1+j, P(1,1,1)=1+i+j, P(0,1,1)=1+i+j, P(1,0,1)=1+j, P(1,1,0)=$ $1+i, P(2,1,1)=1+i+j, P(2,1,0)=1+i, P(0,2,0)=1+i, P(0,2,1)=1+i+j$, $P(1,2,0)=1+i, P(0,0,2)=1+j, P(0,1,2)=1+i+j, P(1,0,2)=1+j, P(1,1,2)=1+i+j$, $P(1,2,1)=1+i+j, P(0,2,2)=1+i+j$ and $P(2,0,1)=1+j$ in which $i^{2}=j^{2}=-1$, forming the numbers in the form $P(n, m, p)$ satisfies the following three-dimensional recurrence conditions:

$$
\left\{\begin{array}{l}
P(n+2, m, p)=P(n, m, p)+P(n-1, m, p) \\
P(n, m+2, p)=P(n, m, p)+P(n, m-1, p) \\
P(n, m, p+2)=P(n, m, p)+P(n, m, p-1)
\end{array}\right.
$$

The tables below represent the coordinate plane of the three-dimensional Padovan relations, for any $n \times m \times p$.

\begin{tabular}{|c|c|c|c|c|c|c|}
\hline $\mathbf{5}$ & $3+2 i$ & $3+2 i$ & $3+2 i$ & $6+4 i$ & $6+4 i$ & $9+6 i$ \\
\hline $\mathbf{4}$ & $2+2 i$ & $2+2 i$ & $2+2 i$ & $4+4 i$ & $4+4 i$ & $6+6 i$ \\
\hline $\mathbf{3}$ & $2+i$ & $2+i$ & $2+i$ & $4+2 i$ & $4+2 i$ & $6+3 i$ \\
\hline $\mathbf{2}$ & $1+i$ & $1+i$ & $1+i$ & $2+2 i$ & $2+2 i$ & $3+3 i$ \\
\hline $\mathbf{1}$ & $1+i$ & $1+i$ & $1+i$ & $2+2 i$ & $2+2 i$ & $3+3 i$ \\
\hline $\mathbf{0}$ & 1 & 1 & 1 & 2 & 2 & 3 \\
\hline $\boldsymbol{n} \times \boldsymbol{m}$ & $\mathbf{0}$ & $\mathbf{1}$ & $\mathbf{2}$ & $\mathbf{3}$ & $\mathbf{4}$ & $\mathbf{5}$ \\
\hline
\end{tabular}

Table 2. Three-dimensional Padovan plane for initial values for $p=0$ 


\begin{tabular}{|c|c|c|c|c|c|c|}
\hline $\mathbf{5}$ & $3+2 i+3 j$ & $3+2 i+3 j$ & $3+2 i+3 j$ & $6+4 i+6 j$ & $6+4 i+6 j$ & $9+6 i+9 j$ \\
\hline $\mathbf{4}$ & $2+2 i+2 j$ & $2+2 i+2 j$ & $2+2 i+2 j$ & $4+4 i+4 j$ & $4+4 i+4 j$ & $6+6 i+6 j$ \\
\hline $\mathbf{3}$ & $2+i+2 j$ & $2+i+2 j$ & $2+i+2 j$ & $4+2 i+4 j$ & $4+2 i+4 j$ & $6+3 i+6 j$ \\
\hline $\mathbf{2}$ & $1+i+j$ & $1+i+j$ & $1+i+j$ & $2+2 i+2 j$ & $2+2 i+2 j$ & $3+3 i+3 j$ \\
\hline $\mathbf{1}$ & $1+i+j$ & $1+i+j$ & $1+i+j$ & $2+2 i+2 j$ & $2+2 i+2 j$ & $3+3 i+3 j$ \\
\hline $\mathbf{0}$ & $1+j$ & $1+j$ & $1+j$ & $2+2 j$ & $2+2 j$ & $3+3 j$ \\
\hline $\boldsymbol{n} \times \boldsymbol{m}$ & $\mathbf{0}$ & $\mathbf{1}$ & $\mathbf{2}$ & $\mathbf{3}$ & $\mathbf{4}$ & $\mathbf{5}$ \\
\hline
\end{tabular}

Table 3. Three-dimensional Padovan plane for initial values for $p=1$

\begin{tabular}{|c|c|c|c|c|c|c|}
\hline $\mathbf{5}$ & $3+2 i+3 j$ & $3+2 i+3 j$ & $3+2 i+3 j$ & $6+4 i+6 j$ & $6+4 i+6 j$ & $9+6 i+9 j$ \\
\hline $\mathbf{4}$ & $2+2 i+2 j$ & $2+2 i+2 j$ & $2+2 i+2 j$ & $4+4 i+4 j$ & $4+4 i+4 j$ & $6+6 i+6 j$ \\
\hline $\mathbf{3}$ & $2+i+2 j$ & $2+i+2 j$ & $2+i+2 j$ & $4+2 i+4 j$ & $4+2 i+4 j$ & $6+3 i+6 j$ \\
\hline $\mathbf{2}$ & $1+i+j$ & $1+i+j$ & $1+i+j$ & $2+2 i+2 j$ & $2+2 i+2 j$ & $3+3 i+3 j$ \\
\hline $\mathbf{1}$ & $1+i+j$ & $1+i+j$ & $1+i+j$ & $2+2 i+2 j$ & $2+2 i+2 j$ & $3+3 i+3 j$ \\
\hline $\mathbf{0}$ & $1+j$ & $1+j$ & $1+j$ & $2+2 j$ & $2+2 j$ & $3+3 j$ \\
\hline $\boldsymbol{n} \times \boldsymbol{m}$ & $\mathbf{0}$ & $\mathbf{1}$ & $\mathbf{2}$ & $\mathbf{3}$ & $\mathbf{4}$ & $\mathbf{5}$ \\
\hline
\end{tabular}

Table 4. Three-dimensional Padovan plane for initial values for $p=2$

Lemma 3.2. The following properties are valid for the Three-dimensional Padovan sequence:

(a) $P(n, 0,0)=P(n)$,

(b) $P(n, 1,1)=P(n)+P(n) i+P(n) j$,

(c) $P(n, 1,0)=P(n)+P(n) i$,

(d) $P(n, 0,1)=P(n)+P(n) j$.

Proof. To demonstrate the property $P(n, 0,0)=P(n)$, the relation $P(n+2, m, p)=P(n, m, p)+$ $P(n-1, m, p)$ will be considered and the initials values obtained by empirical means $P(0,0,0)=$ $1=P(0), P(1,0,0)=1=P(1), P(2,0,0)=1=P(2)$. Thus, for $m=p=0$, that is, $P(n+2,0,0)=P(n, 0,0)+P(n-1,0,0)$, varying $n=(0,1,2,3, \ldots)$. It can be seen that:

$$
\begin{aligned}
P(n+2,0,0) & =P(n, 0,0)+P(n-1,0,0) ; \\
P(3,0,0) & =P(1,0,0)+P(0,0,0)=2=P(3) ; \\
P(4,0,0) & =P(2,0,0)+P(1,0,0)=2=P(4) ; \\
P(5,0,0) & =P(3,0,0)+P(2,0,0)=3=P(5) ; \\
P(6,0,0) & =P(4,0,0)+P(3,0,0)=4=P(6) ; \\
& \vdots \\
P(n-3,0,0) & =P(n-5,0,0)+P(n-6,0,0)=P(n-3) ; \\
P(n-2,0,0) & =P(n-4,0,0)+P(n-5,0,0)=P(n-2) ; \\
P(n-1,0,0) & =P(n-3,0,0)+P(n-4,0,0)=P(n-1) ; \\
P(n, 0,0) & =P(n-2,0,0)+P(n-3,0,0) \\
& =P(n-2)+P(n-3)=P(n) .
\end{aligned}
$$

The other identities are demonstrated similarly to identity (a). 
Lemma 3.3. The following properties are valid:

(a) $P(0, m, 0)=P(m)+P(m-1) i$,

(b) $P(0, m, 1)=P(m)+P(m-1) i+P(m) j$,

(c) $P(1, m, 0)=P(m)+P(m-1) i$,

(d) $P(1, m, 1)=P(m)+P(m-1) i+P(m) j$.

Proof. From recurrence $P(n, m+2, p)=P(n, m, p)+P(n, m-1, p)$ and with initial values $P(0)=P(1)=P(2)=1, P(0,0,0)=1, P(0,1,0)=1+i$ and $P(0,2,0)=1+i$, the second induction principle can be applied to $m$ in $n=p=0$. Thus, varying $m=1,2,3, \ldots, k$, we realize that:

$$
\begin{aligned}
P(0, m+2,0) & =P(0, m, 0)+P(0, m-1,0) ; \\
P(0,3,0) & =P(0,1,0)+P(0,0,0)=2+i=P(3)+P(2) i ; \\
P(0,4,0) & =P(0,2,0)+P(0,1,0)=2+2 i=P(4)+P(3) i ; \\
P(0,5,0) & =P(0,3,0)+P(0,2,0)=3+2 i=P(5)+P(4) i ; \\
P(0,6,0) & =P(0,4,0)+P(0,3,0)=4+3 i=P(6)+P(5) i ; \\
& \vdots \\
P(0, k-3,0) & =P(0, k-5,0)+P(0, k-6,0)=P(k-3)+P(k-4) i ; \\
P(0, k-2,0) & =P(0, k-4,0)+P(0, k-5,0)=P(k-2)+P(k-3) i ; \\
P(0, k-1,0) & =P(0, k-3,0)+P(0, k-4,0)=P(k-1)+P(k-2) i ; \\
P(0, k, 0) & =P(0, k-2,0)+P(0, k-3,0) \\
& =P(k-2)+P(k-3) i+P(k-3)+P(k-4) i \\
& =P(k)+P(k-1) i .
\end{aligned}
$$

Demonstrating property $P(0, m, 0)=P(m)+P(m-1) i$.

The other identities are demonstrated similarly to identity (a).

Lemma 3.4. The following identities are valid:

(a) $P(0,0, p)=P(p)+P(p-1) j$,

(b) $P(0,1, p)=P(p)+P(p) i+P(p-1) j$,

(c) $P(1,0, p)=P(p)+P(p-1) j$,

(d) $P(1,1, p)=P(p)+P(p) i+P(p-1) j$.

Proof. Being $P(n, m, p+2)=P(n, m, p)+P(n, m, p-1)$ with the initial values $P(0)=$ $P(1)=P(2)=1, P(0,0,0)=1, P(0,0,1)=1+j, P(0,0,2)=1+j$ and, applying the second principle of induction $p$ for $n=m=0$ and varying $p=1,2,3, \ldots, k$, we have to:

$$
\begin{aligned}
P(0,0, p+2) & =P(0,0, p)+P(0,0, p-1) ; \\
P(0,0,3) & =P(0,0,1)+P(0,0,0)=2+j=P(3)+P(2) j ; \\
P(0,0,4) & =P(0,0,2)+P(0,0,1)=2+2 j=P(4)+P(3) j ; \\
P(0,0,5) & =P(0,0,3)+P(0,0,2)=3+2 j=P(5)+P(4) j ; \\
P(0,0,6) & =P(0,0,4)+P(0,0,3)=4+3 j=P(6)+P(5) j ;
\end{aligned}
$$




$$
\begin{aligned}
P(0,0, k-3) & =P(0,0, k-5)+P(0,0, k-6)=P(k-3)+P(k-4) j ; \\
P(0,0, k-2) & =P(0,0, k-4)+P(0,0, k-5)=P(k-2)+P(k-3) j ; \\
P(0,0, k-1) & =P(0,0, k-3)+P(0,0, k-4)=P(k-1)+P(k-2) j ; \\
P(0,0, k) & =P(0,0, k-2)+P(0,0, k-3) \\
& =P(k-2)+P(k-3) j+P(k-3)+P(k-4) j \\
& =P(k)+P(k-1) j .
\end{aligned}
$$

Validating the property $P(0,0, p)=P(p)+P(p-1) j$.

The other identities are demonstrated similarly to identity (a).

Theorem 3.5. For $n, m, p \in \mathbb{N}$, the numbers in the form $P(n, m, p)$ are described by:

$$
P(n, m, p)=P(n) P(m) P(p)+P(n) P(m-1) P(p) i+P(n) P(m) P(p-1) j .
$$

Proof. So for $m=2$ with $P(0,2,0)=1+i, P(0,2,1)=1+i+j, P(0,2,2)=1+i+j$, it has to be:

$$
\begin{aligned}
P(0,2,3) & =P(0,2,1)+P(0,2,0)=2+2 i+j=P(3)+P(3) i+P(2) j ; \\
P(0,2,4) & =P(0,2,2)+P(0,2,1)=2+2 i+2 j=P(4)+P(4) i+P(3) j ; \\
P(0,2,5)= & P(0,2,3)+P(0,2,2)=3+3 i+2 j=P(5)+P(5) i+P(4) j ; \\
P(0,2,6)= & P(0,2,4)+P(0,2,3)=4+4 i+3 j=P(6)+P(6) i+P(5) j ; \\
& \vdots \\
P(0,2, p-3)= & P(0,2, p-5)+P(0,2, p-6)=P(p-3)+P(p-3) i+P(p-4) j ; \\
P(0,2, p-2)= & P(0,2, p-4)+P(0,2, p-5)=P(p-2)+P(p-2) i+P(p-3) j ; \\
P(0,2, p-1)= & P(0,2, p-3)+P(0,2, p-4)=P(p-1)+P(p-1) i+P(p-2) j ; \\
P(0,2, p)= & P(0,2, p-2)+P(0,2, p-3) \\
= & P(p-2)+P(p-2) i+P(p-3) j \\
& +P(p-3)+P(p-3) i+P(p-4) j \\
= & P(p)+P(p) i+P(p-1) j .
\end{aligned}
$$

Then, for $n=1,2,3, \ldots, m$ we have that:

$$
\begin{aligned}
& P(0,0, p)=P(0) P(p)+P(-1) P(p) i+P(0) P(p-1) j ; \\
& P(0,1, p)=P(1) P(p)+P(0) P(p) i+P(1) P(p-1) j ; \\
& P(0,2, p)=P(2) P(p)+P(1) P(p) i+P(2) P(p-1) j ; \\
& P(0,3, p)=P(3) P(p)+P(2) P(p) i+P(3) P(p-1) j ; \\
& P(0,4, p)=P(4) P(p)+P(3) P(p) i+P(4) P(p-1) j ; \\
& P(0,5, p)=P(5) P(p)+P(4) P(p) i+P(5) P(p-1) j ;
\end{aligned}
$$$$
P(0, k-3, p)=P(0, k-5, p)+P(0, k-6, p)=P(k-3) P(p)+(P(k-4) P(p)) i
$$$$
+(P(k-3) P(p-1)) j \text {; }
$$ 


$$
\begin{aligned}
P(0, k-1, p)= & P(0, k-3, p)+P(0, k-2, p)=P(k-1) P(p)+(P(k-2) P(p)) i \\
& +(P(k-1) P(p-1)) j ; \\
P(0, k, p)= & P(0, k-2, p)+P(0, k-3, p) \\
= & P(k-2) P(p)+(P(k-3) P(p)) i+(P(k-2) P(p-1)) j ; \\
& +P(k-3) P(p)+(P(k-4) P(p)) i+(P(k-3) P(p-1)) j ; \\
= & P(k) P(p)+(P(k-1) P(p)) i+(P(k) P(p-1)) j .
\end{aligned}
$$

This proves the veracity of the Theorem 3.5 , by applying it to $n=1,2,3, \ldots, k$, in the following situation:

$$
\begin{aligned}
P(1, m, p)= & P(1) P(m) P(p)+P(1) P(m-1) P(p) i+P(1) P(m) P(p-1) j ; \\
P(2, m, p)= & P(2) P(m) P(p)+P(2) P(m-1) P(p) i+P(2) P(m) P(p-1) j ; \\
P(3, m, p)= & P(3) P(m) P(p)+P(3) P(m-1) P(p) i+P(3) P(m) P(p-1) j ; \\
P(4, m, p)= & P(4) P(m) P(p)+P(4) P(m-1) P(p) i+P(4) P(m) P(p-1) j ; \\
P(5, m, p)= & P(5) P(m) P(p)+P(5) P(m-1) P(p) i+P(5) P(m) P(p-1) j ; \\
P(6, m, p)= & P(6) P(m) P(p)+P(6) P(m-1) P(p) i+P(6) P(m) P(p-1) j ; \\
\vdots & \\
P(k-3, m, p)= & P(k-3) P(m) P(p)+P(k-3) P(m-1) P(p) i \\
& +P(k-3) P(m) P(p-1) j ; \\
P(k-2, m, p)= & P(k-2) P(m) P(p)+P(k-2) P(m-1) P(p) i \\
& +P(k-2) P(m) P(p-1) j ; \\
P(k-1, m, p)= & P(k-1) P(m) P(p)+P(k-1) P(m-1) P(p) i \\
& +P(k-1) P(m) P(p-1) j ; \\
P(k, m, p)= & P(k-2, m, p)+P(k-3, m, p) \\
= & P(k-2) P(m) P(p)+P(k-2) P(m-1) P(p) i \\
& +P(k-2) P(m) P(p-1) j+P(k-3) P(m) P(p) \\
& +P(k-3) P(m-1) P(p) i+P(k-3) P(m) P(p-1) j \\
= & P(k) P(m) P(p)+P(k) P(m-1) P(p) i \\
& +P(k) P(m) P(p-1) j .
\end{aligned}
$$

\section{On higher dimensional for the Padovan sequence recurrence}

Based on the work of Harman [7], Oliveira, Alves and Paiva [12] and Polatli [15] the recurrent on higher dimensional relations of the Padovan sequence will be defined, thus designating a generalized expression for the hypercomplex numbers described in the form $P\left(n_{1}, n_{2}, n_{3}, \ldots, n_{n}\right)$, where $n$ represents each dimensional variable. The hypercomplex numbers are defined in the form $a_{0}+a_{1} i_{1}+a_{2} i_{2}+\cdots+a_{n} i_{n}$ where $a_{0}, a_{1}, a_{2}, \ldots, a_{n}$ represent an arbitrary real number and $i_{1}, i_{2}, \ldots, i_{n}$ are certain symbols that represent the imaginary units introduced by Kantor [10]. 
Definition 4.1. A number on higher dimensional of Padovan $P\left(n_{1}, n_{2}, n_{3}, \ldots, n_{n}\right)$, with $n$ variables and $n_{1}, n_{2}, n_{3}, \ldots, n_{n} \in \mathbb{N}$ for $n \geqslant 3$, and the following set of imaginary units $\left(\mu_{1}=i, \mu_{2}=j, \ldots, \mu_{n}\right)$. So, the following initial values are defined:

$$
\begin{aligned}
P(0,0,0, \ldots, 0) & =0 ; \\
P(1,0,0, \ldots, 0) & =1 ; \\
P(0,1,0, \ldots, 0) & =\mu_{1} ; \\
P(0,0,1, \ldots, 0) & =\mu_{2} ; \\
P(0,0,0,1, \ldots, 0) & =\mu_{3} ; \\
P(0,0,0,0,1,0, \ldots, 0) & =\mu_{4} ; \\
& \vdots \\
P(0,0,0, \ldots, 1) & =\mu_{n-1} ; \\
P(1,1,1, \ldots, 1) & =1+i+\mu_{1}+\ldots+\mu_{n} ; \\
P(0,1,1, \ldots, 1) & =i+\mu_{1}+\ldots+\mu_{n} ; \\
P(1,0,1, \ldots, 1) & =1+\mu_{1}+\ldots+\mu_{n} ; \\
P(1,1,0, \ldots, 1) & =1+i+\mu_{2}+\ldots+\mu_{n} ; \\
& \vdots \\
P(1,1,1, \ldots, 1,0) & =1+\mu_{1}+\mu_{2}+\ldots+\mu_{n-1} .
\end{aligned}
$$

Thus, the numbers in the form $P\left(n_{1}, n_{2}, n_{3}, \ldots, n_{n}\right)$ satisfy the following on higher dimensional recurrence conditions:

$$
\begin{aligned}
P\left(n_{1}, n_{2}, n_{3}, \ldots, n_{n}\right) & =P\left(n_{1}-2, n_{2}, n_{3}, \ldots, n_{n}\right)+P\left(n_{1}-3, n_{2}, n_{3}, \ldots, n_{n}\right) ; \\
P\left(n_{1}, n_{2}, n_{3}, \ldots, n_{n}\right) & =P\left(n_{1}, n_{2}-2, n_{3}, \ldots, n_{n}\right)+P\left(n_{1}, n_{2}-3, n_{3}, \ldots, m_{n}\right) ; \\
P\left(n_{1}, n_{2}, n_{3}, \ldots, n_{n}\right) & =P\left(n_{1}, n_{2}, n_{3}-2, \ldots, n_{n}\right)+P\left(n_{1}, n_{2}, n_{3}-3, \ldots, n_{n}\right) ; \\
& \vdots \\
P\left(n_{1}, n_{2}, n_{3}, \ldots, n_{n}\right) & =P\left(n_{1}, n_{2}, n_{3}, \ldots, n_{n}-2\right)+P\left(n_{1}, n_{2}, n_{3}, \ldots, n_{n}-3\right) .
\end{aligned}
$$

Theorem 4.2. The numbers in the form $P\left(n_{1}, n_{2}, n_{3}, \ldots, n_{n}\right)$, such that $n_{1}, n_{2}, n_{3}, \ldots, n_{n} \in \mathbb{N}$, are determined by:

$$
\begin{aligned}
P\left(n_{1}, n_{2}, n_{3}, \cdots, n_{n}\right)= & \left(P\left(n_{1}\right) P\left(n_{2}\right) P\left(n_{3}\right) \cdots P\left(n_{n}\right)\right) \\
& +\left(P\left(n_{1}\right) P\left(n_{2}-1\right) P\left(n_{3}\right) \cdots P\left(n_{n}\right)\right) \mu_{1} \\
& +\left(P\left(n_{1}\right) P\left(n_{2}\right) P\left(n_{3}-1\right) \cdots P\left(n_{n}\right)\right) \mu_{2} \\
& +\cdots \\
& +\left(P\left(n_{1}\right) P\left(n_{2}\right) P\left(n_{3}\right) \cdots P\left(n_{n}-1\right)\right) \mu_{n} .
\end{aligned}
$$

Proof. Based on the statements made in the previous subsections, where the theorems are valid $P(n, m)=P(n) P(m)+P(n) P(m-1) i$ and $P(n, m, p)=P(n) P(m) P(p)+P(n) P(m-1)$ $P(p) i+P(n) P(m) P(p-1) j$, by means of induction we can verify that: 


$$
\begin{aligned}
P(n, m)= & P(n) P(m)+P(n) P(m-1) i \\
P(n, m, p)= & P(n) P(m) P(p)+P(n) P(m-1) P(p) i \\
& +P(n) P(m) P(p-1) j \\
P(n, m, p, q)= & P(n) P(m) P(p) P(q)+P(n) P(m-1) P(p) P(q) i \\
& +P(n) P(m) P(p-1) P(q) j+P(n) P(m) P(p) P(q-1) k ; \\
\vdots & \left(P\left(n_{1}\right) P\left(n_{2}\right) P\left(n_{3}\right) \cdots P\left(n_{n}\right)\right) \\
P\left(n_{1}, n_{2}, n_{3}, \ldots, n_{n}\right)= & \left(P\left(n_{1}\right) P\left(n_{2}-1\right) P\left(n_{3}\right) \cdots P\left(n_{n}\right)\right) \mu_{1} \\
& +\left(P\left(n_{1}\right) P\left(n_{2}\right) P\left(n_{3}-1\right) \cdots P\left(n_{n}\right)\right) \mu_{2} \\
& +\cdots \\
& +\left(P\left(n_{1}\right) P\left(n_{2}\right) P\left(n_{3}\right) \cdots P\left(n_{n}-1\right)\right) \mu_{n} .
\end{aligned}
$$

\section{Conclusion}

A discussion about the Padovan sequence complexification process was presented in this paper. This was done using inserting an imaginary unit based on the works of Oliveira, Alves and Paiva [12], Alves and Catarino [2], in which they carry out the complexification process for the Fibonacci sequence, being possible to carry out the same process but for another sequence. Thus, a whole new set of algebraic representations and properties are developed for Padovan sequence.

The two-dimensional and three-dimensional recurrent relations are explored in order to understand and describe the Padovan sequence generalization through mathematical properties.

The complexation process was generalized for an $n$-dimensional complex Padovan sequence supported by Oliveira, Alves, and Paiva [12] and Alfred [1] and Horadam [8], where they present similar relations for Fibonacci numbers and their properties.

For future work, one can explore the application and alternative representations of these numbers in computational fields, such as graphs and geometrical representations. Other properties and identities like the sum of powers of terms of a linear recurrence sequence as performed by Chaves and Marques [5], Klavzar and Mollard [11].

\section{Acknowledgements}

Part of the development of research in Brazil had the financial support of the National Council for Scientific and Technological Development (CNPq) and a Coordination for the Improvement of Higher Education Personnel (CAPES). The research development part in Portugal is financed by National Funds through the Foundation for Science and Technology. I. P (FCT), under the project UID/CED/00194/2020. 


\section{References}

[1] Alfred, B. U. (1965). An Introduction to Fibonacci Discovery. The Fibonacci Association Cleveland, Cleveland, OH, U.S.A.

[2] Alves, F. R. V., \& Catarino, P. M. M. C. (2017). A classe dos polinômios bivariados de Fibonacci (PBF): elementos recentes sobre a evolução de um modelo. Revista Thema, 14(1), $112-136$.

[3] Bilgici, G. (2017). Fibonacci and Lucas Sedenions. Journal of Integer Sequences, 20, 1-11.

[4] Catarino, P. M. M. C. (2018). Diagonal Functions of the $k$-Pell and $k$-Pell-Lucas Polynomials and some identities. Acta Mathematica Universitatis Comenianae, 87(1), 147-159.

[5] Chaves, A. P., Marques, D., \& Togbé, A. (2012). On the sum of powers of terms of a linear recurrence sequence, Bulletin of the Brazilian Mathematical Society, 43(3), 397-406.

[6] Claudi, A., \& Nelsen, R. B. (2015). A mathematical space odyssey: solid geometry in the 21th Century, American Mathematical Society; Edição: UK ed., Washington.

[7] Harman, C. J. (1981). Complex fibonacci numbers, The Fibonacci Quarterly, 19, 82-86.

[8] Horadam, A. F. (1993). Quaternion recurrence relations. Ulam Quarterly, 2(2), 23-33.

[9] Jafari, M. (2016). On the Matrix Algebra of Complex Quaternions. Preprint. DOI: 10.13140/RG.2.1.3565.2321.

[10] Kantor, I. L., \& Solodovnikov, A. S. (1989). Hypercomplex Numbers - An Elementary Introduction to Algebras Translated by A. Shenitzer, The Fibonacci Association Cleveland, Springer-Verlag, New York.

[11] Klavzar, S., \& Mollard, M. (2012). Cube Polynomial of Fibonacci and Lucas Cubes. Acta Applicandae Mathematicae, 117, 93-105.

[12] Oliveira, R. R. de, Alves, F. R. V., \& Paiva, R. E. B. (2017). Identidades bi e tridimensionais para os números de Fibonacci na forma complexa. C.Q.D.-Revista Eletrônica Paulista de Matemática, 11, 91-106.

[13] Özdemir, G., Simsek, Y., \& Milovanović, G. V. (2017). Generating Functions for Special Polynomials and Numbers Including Apostol-Type and Humbert-Type Polynomials. Mediterranean Journal of Mathematics, 14(117), 1-16.

[14] Padovan, R. (2002). Dom Hans van der Laan and the plastic number. Nexus Network Journal, 4, 181-193.

[15] Polatl1, E., \& Kesim, S. (2015). On quaternions with generalized Fibonacci and Lucas number components. Advances in Difference Equations, 169, 1-8. 
[16] Savin, D. (2015). Some properties of Fibonacci numbers, Fibonacci octonions, and generalized Fibonacci-Lucas octonions, Advances in Difference Equations, 298, 1-10.

[17] Stewart, I. (1996). Tales of a Neglected Number. Mathematical Recreations - Scientific American, 274(6), 102-103.

[18] Voet, C., \& Schoonjans, Y. (2012). Benedictine thought as a catalist for 20th century liturgical space: the motivation behind dom hans van der laan s aesthetic church arquitectury, Proceeding of the 2nd international conference of the Europa Architetural History of Network, 255-261.

[19] Yilmaz, N., \& Taskara, N. (2016). On the Properties of Iterated Binomial Transforms for the Padovan and Perrin Matrix Sequences. Mediterranean Journal of Mathematics, 13, $1435-1447$. 